\title{
Erectile dysfunction severity and SYNTAX score in coronary artery disease
}

\begin{abstract}
Introduction: Erectile dysfunction (ED) can be an early manifestation of coronary artery disease (CAD). The SYNTAX score (SS) is used in grading CAD complexity by evaluating lesions on angiography. The present study aimed to investigate the relationship between ED severity and SS in patients with CAD who underwent coronary angiography.

Materials and methods: Male patients undergoing coronary angiography due to coronary artery stenosis were included. Angiograms of the patients were scored into three groups regarding the SS: low ( $\mathrm{SS} \leq 22$ ), intermediate ( $\mathrm{SS}$ between 23-32), and high ( $\mathrm{SS} \geq 33$ ). Erectile performances were evaluated by the International Index of Erectile Function (IIEF). The patients were divided into 4 groups regarding IIEF scores: severe (IIEF: 0-10), moderate ED (IIEF: 11-16), mild (IIEF: 17-25), and no (IIEF: 26-30). Patients' data regarding age, body mass index, smoking, diabetes, hypertension, and blood lipid profiles were recorded.
\end{abstract}

Results: Of 58 patients, $13(22.4 \%)$ were in the low SS, $29(50.0 \%)$ were in the moderate SS, and $16(27.6 \%)$ were in the high SS group. Mild ED was present in $96.6 \%(n=56)$ of the patients. There were significant differences among the SS groups regarding the IIEF scores; the IIEF score significantly decreased as the SS score increased. In the 43-59-year age group, the IIEF score was moderate in the low SS group and significantly higher than those in the high SS group.

Conclusion: The degree of ED increased with the increase in SS. Using the SS was beneficial in demonstrating the severity of CAD and ED.

Keywords: erectile dysfunction, coronary artery disease, syntax score, coronary angiography
Volume 8 Issue 5 - 2017

\author{
Mustafa Bilal Tuna,' Kenan Abdurrahman \\ Kara, ${ }^{2}$ Omer Burak Argun,' Tansu Gudelci, \\ Egemen Duygu, ${ }^{2}$ Murat Sener ${ }^{3}$ \\ 'Department of Urology,Ac?badem Aile Hospital,Turkey \\ ${ }^{2}$ Department of Cardiology, Ac?badem Aile Hospital, Turkey \\ ${ }^{3}$ Department of Cardiovascular Surgery, Medicana
}

Correspondence: Kenan Abdurrahman Kara, Aile Hastanesi Kalp Damar Cerrahisi Bölümü, Bahçelievler Mh., Talatpa?a Bulv. Begonya Sok. No:7/9, 34|80, Bahçelievler, Istanbul, Turkey, Tel +90530 0655225, Fax +90212 44I1300 Email kenankaradoc@gmail.com

Received: April 17, 2017| Published: April 21, 2017

\section{Introduction}

Erectile dysfunction(ED) is common in patients with cardiovascular disease (CVD). ED is an important component of quality of life as well as an independent indicator of future cardiovascular events. ${ }^{1}$ CVD is an important cause of morbidity and mortality accounting for $30 \%$ of overall deaths worldwide. ${ }^{2}$ Besides, CVD comprises many risk factors for sexual dysfunction. In individuals with CVD, pathological processes influencing vascular configurations also influence the arteries that provide blood supply to the genital organs and lead to high rates of sexual dysfunction both in males and females. ${ }^{3}$ In the European Association of Urology (EAU) guidelines, ED is defined as "the persistent inability to attain and maintain an erection sufficient to permit satisfactory sexual performance". ${ }^{4}$ The causes of ED can be vasculogenic, neurogenic, anatomical or structural, hormonal, druginduced, and psychogenic. ${ }^{4}$ In a study evaluating 948 patients with ED, organic etiology was determined in $34.6 \%$ of the patients, of whom $40.5 \%$ had arteriogenic ED. ${ }^{5}$ Functional vascular ED, which is an impairment in endothelial-dependent or -independent smooth muscle relaxation, or structural vascular $\mathrm{ED}$, which is the penile artery occlusions due to atherosclerosis, or functional and structural vascular ED together can lead to vasculogenic ED. ${ }^{6}$

Prevalence rate of ED, which shows variation among the methods used, age groups, and socioeconomic and cultural structures, ranges between $10 \%$ and $60 \%{ }^{4}$ Although ED is considered as a benign disorder, it may be an early manifestation of coronary artery disease (CAD) and peripheral vascular disease. In principle, the risk factors of ED and CVD (such as age, weight, hypertension, diabetes, hyperlipidemia, and cigarette smoking) are similar to each other. Thus, patients with even mild ED should be evaluated for underlying CVD. The Princeton III consensus ${ }^{7}$ recommends that all patients with $\mathrm{ED}$ who are older than 30years have an increased risk for CAD and should be evaluated for CVD status through a non-invasive way and, when indicated, through an invasive way.

The SYNTAX score (SS) is a recently defined scoring system used in grading complexity of CAD by evaluating the lesions on angiography. ${ }^{8}$ Using the SS is beneficial both for objective risk stratification in patients and for providing optimal revascularization strategy by enhancing collaboration between a surgeon and a cardiologist. ${ }^{9}$ It has been reported that ED is present in $46 \%$ of males with CAD and that there is a correlation between severity of CAD and $\mathrm{ED} 1^{3}$ The aim of the present study was to investigate the relationship between ED severity and SS in patients with $\mathrm{CAD}$ who underwent coronary angiography.

\section{Materials and methods}

Male patients who underwent coronary angiography due to coronary artery stenosis were included. Patients who were using drugs chronically (except ACE inhibitors, beta blockers, calcium channel blockers, diuretics, nitrates, oral antidiabetics, insulin, statins, and acetyl salicylic acid) and who had a history of previous pelvic urologic surgery, neurologic disease, or endocrinologic and psychiatric disorder were excluded from the study. None of the patients were on the treatment for ED. All of the patients in the present study were married or had a regular partner. Written informed contest was obtained from each patient. 
Angiograms of the patients were scored and divided into three groups according to the SS as low ( $\mathrm{SS} \leq 22$ ), intermediate (SS between 23-32), and high (SS $\geq 33){ }^{8}$ After coronary angiography, erectile performance statuses of all patients were evaluated using the International Index of Erectile Function (IIEF), which is a validated 15-item self-administered questionnaire. ${ }^{10}$ IEFF was translated and validated into Turkish. ${ }^{11}$ The patients were divided into 4 groups based on their IIEF scores as severe ED (IIEF score: 0-10), moderate ED (IIEF score: 11-16), mild ED (IIEF score: 17-25), and no ED (IIEF score: 26-30).

Data of the patients regarding age, body mass index, smoking, presence of diabetes mellitus (defined as a fasting glucose level of $\geq 126 \mathrm{mg} / \mathrm{dL}$ in two analyses) and hypertension (defined as blood pressure $\geq 140 / 90 \mathrm{mmHg}$ in consecutive measurements), and blood lipid profiles (total cholesterol, high density lipoprotein, low density lipoprotein, and triglycerides) were recorded.

\section{Statistical analysis}

PASW 18.0 for Windows program (SPSS Inc., Chicago, IL, USA) was used for statistical analyses. Multiple group comparison for numerical variables was performed using ANOVA test in case assumption of normal distribution was provided, whereas KruskalWallis test was used when assumption of normal distribution was not provided. Comparison of two independent groups for numerical variables was performed by Mann-Whitney $U$ test when assumption of normal distribution was not provided. Mann-Whitney U test with Bonferroni correction was used for subgroup comparisons for nonnormally distributed variables. Comparison of multiple groups for categorical variables was performed by chi-square test when the condition was met, whereas Monte Carlo Simulation was used when the condition was not met. The level of statistical significance was considered as $\mathrm{p}<0.05$.

\section{Results}

Of 58 patients enrolled into the study, $13(22.4 \%)$ were in the low SS, $29(50.0 \%)$ were in the moderate SS, and $16(27.6 \%)$ were in the high SS group. ED was not present in $3.4 \%(\mathrm{n}=2)$ of the patients, whereas $96.6 \%(n=56)$ had mild ED. Owing to inadequate number of patients, ED groups could not be compared in terms of risk factors. Comparison among the SS groups for risk factors revealed no difference in terms of age, body mass index, total cholesterol, high density lipoprotein, low density lipoprotein and triglyceride levels, smoking, presence of diabetes, and presence of hypertension (Table 1).

There were significant differences among the SS groups in terms of the IIEF scores (Table 2). The IIEF score significantly decreased as the SS score increased. In order to evaluate the effect of age on the IIEF scores, comparisons according to the age groups were performed among the SS groups. In the 43-59-year age group, the IIEF score was moderate in the low SS group and significantly higher as compared to the high SS group. This was also similar in the other age groups; however, a statistically significant difference could not be demonstrated because of the limited number of patients (Table 3 ).

On the other hand, there were no significant differences between the IIEF scores of the patients with and without diabetes among the SS groups (Table 4). There were also no significant differences between the IIEF scores of the patients with and without hypertension among the SS groups (Table 4). No significant differences were determined between the IIEF scores of smokers and non-smokers among the SS groups (Table 4).

Table I Comparison of the Syntax Score groups for the risk factors

\begin{tabular}{|c|c|c|c|c|}
\hline & Low SS Group $(n=\mid 3)$ & Moderate SS Group $(n=29)$ & High SS Group $(n=16)$ & $\mathbf{p}$ \\
\hline Age, year & $59.23 \pm 9.04(57)$ & $60.55 \pm 9.31(59)$ & $62.69 \pm 8.78(59.5)$ & 0.543 \\
\hline $\mathrm{BMI}, \mathrm{kg} / \mathrm{m} 2$ & $30.46 \pm 2.73(31)$ & $29.72 \pm 2.45(30)$ & $29.36 \pm 2.92(29.5)$ & 0.45 \\
\hline Total cholesterol, mg/dL & $188.08 \pm 12.63(184)$ & $189.48 \pm 13.73(190)$ & $191.88 \pm 4.41$ (191.5) & 0.24 \\
\hline $\mathrm{HDL}, \mathrm{mg} / \mathrm{dL}$ & $42.15 \pm 6.43(42)$ & $42.24 \pm 5.23(4 I)$ & $39.38 \pm 5.37(40.5)$ & 0.368 \\
\hline $\mathrm{LDL}, \mathrm{mg} / \mathrm{dL}$ & $111.99 \pm 13.48(|1| 1.6)$ & II $2.24 \pm \mid 4.72($ (II I.4) & $116.24 \pm 9.95(117.4)$ & 0.167 \\
\hline Triglyceride, mg/dL & $169.69 \pm 20.46(162)$ & $|74.72 \pm| 7.6 \mid(\mid 72)$ & $181.31 \pm 16.64(177)$ & 0.225 \\
\hline Presence of diabetes mellitus & $3(23.1)$ & $6(20.7)$ & $5(31.3)$ & 0.73 \\
\hline Presence of hypertension & $4(30.8)$ & $15(5 \mid .7)$ & $9(56.3)$ & 0.343 \\
\hline Smoking & $8(6 \mid .5)$ & $10(34.5)$ & $6(37.5)$ & $0.24 I$ \\
\hline
\end{tabular}

Values are presented as mean \pm standard deviation (median) or number (\%), where appropriate.

SS: SYNTAX Score; BMI: Body Mass Index; HDL: High Density Lipoprotein; LDL: Low Density Lipoprotein.

Table 2 The International Index of Erectile Function scores the Syntax Score groups

\begin{tabular}{lllll}
\hline & Low SS Group $(\mathbf{n}=\mathbf{1 3})$ & Moderate SS Group $(\mathbf{n}=\mathbf{2 9})$ & High SS Group $(\mathbf{n}=\mathbf{1 6})$ & $\mathbf{p} *$ \\
\hline \multirow{3}{*}{ Mean $\pm S D($ Median$)$} & Mean $\pm S D($ Median$)$ & Mean $\pm S D($ Median$)$ & \\
\hline
\end{tabular}

${ }^{\mathrm{a}}$ Different from the low SS group, ${ }^{\mathrm{b}}$ Different from the moderate SS group, ${ }^{\mathrm{c} D i f f e r e n t}$ from the high SS group. * Mann-Whitney $\mathrm{U}$ test with Bonferroni correction was used ( $<$ <0.017). SS: SYNTAX Score; SD: Standard deviation; IIEF: International Index of Erectile Function.

Table 3 The International Index of Erectile Function scores the Syntax Score groups according to age groups

\begin{tabular}{|c|c|c|c|c|c|c|}
\hline \multirow[t]{3}{*}{ Age Groups,Year } & \multicolumn{6}{|c|}{ IIEF scores } \\
\hline & \multicolumn{2}{|c|}{ Low SS Group } & \multicolumn{2}{|c|}{ Moderate SS Group } & \multicolumn{2}{|c|}{ High SS Group } \\
\hline & $\mathbf{n}$ & Mean $\pm S D$ (Median) & $\mathbf{n}$ & Mean $\pm S D$ (Median) & $\mathbf{n}$ & Mean $\pm S D$ (Median) \\
\hline $43-59$ & 7 & $24.00 \pm 0.82(24) b, c$ & 15 & $20.47 \pm \mathrm{I} .77(2 \mathrm{I}) \mathrm{a}$ & 8 & $19.13 \pm 0.83(19) a$ \\
\hline $60-69$ & 4 & $25.00 \pm 0.82(25)$ & 7 & $20.7 I \pm I .98(2 I)$ & 5 & $19.60 \pm 0.89$ (19) \\
\hline$>70$ & 2 & $24.50 \pm 2.12(24.5)$ & 7 & $21.57 \pm 1.13(21)$ & 3 & $20.67 \pm 0.58(21)$ \\
\hline
\end{tabular}

aDifferent from the low SS group, bDifferent from the moderate SS group, cDifferent from the high SS group. *Mann-Whitney $U$ test with Bonferroni correction was used $(p<0.017)$ 
Table 4 The International Index of Erectile Function scores among the Syntax Score groups according to presence/absence of diabetes and hypertension and smoking

\begin{tabular}{|c|c|c|c|c|}
\hline \multicolumn{5}{|c|}{ IIEF Scores } \\
\hline & $\mathbf{n}$ & Non-diabetics & $\mathbf{n}$ & Diabetics \\
\hline Low SS group & 10 & $24.50 \pm 1.08(24.5)$ & 3 & $24.00 \pm 1.00(24)$ \\
\hline Moderate SS group & 23 & $20.74 \pm 1.89(21)$ & 6 & $21.00 \pm 0.63(21)$ \\
\hline \multirow[t]{2}{*}{ High SS group } & II & 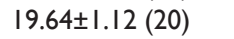 & 5 & $19.40 \pm 0.55$ (19) \\
\hline & $\mathrm{n}$ & Non-hypertensives & $\mathrm{n}$ & Hypertensives \\
\hline Low SS group & 9 & $24.44 \pm 0.88(24)$ & 4 & $24.25 \pm 1.50(24)$ \\
\hline Moderate SS group & 14 & $20.64 \pm 2.24(20)$ & 15 & $20.93 \pm 1.03(21)$ \\
\hline \multirow[t]{2}{*}{ High SS group } & 7 & $19.86 \pm 1.22(20)$ & 9 & 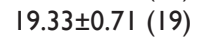 \\
\hline & $\mathrm{n}$ & Non-smokers & $\mathrm{n}$ & Smokers \\
\hline Low SS group & 5 & $24.40 \pm 1.14(24)$ & 8 & $24.38 \pm 1.06(24.5)$ \\
\hline Moderate SS group & 19 & $20.84 \pm I .26(2 I)$ & 10 & $20.70 \pm 2.4 \mathrm{I}(20.5)$ \\
\hline High SS group & 10 & $19.70 \pm 0.95(20)$ & 6 & $19.33 \pm 1.03(19)$ \\
\hline
\end{tabular}

*Analysis could not be performed because of the limited number of patients.

Data are presented as mean \pm standard deviation (median).

IIEF: International Index of Erectile Function; SS: SYNTAX Score.

\section{Discussion}

Erectile dysfunction and CVD have similar risk factors such as hypertension, dyslipidemia, diabetes, depression, obesity, and cigarette smoking. ${ }^{12}$ The relationship between ED and CVD has been proven both pathophysiologically and clinically. ${ }^{13,14}$ Salem et al., ${ }^{15}$ suggested that ED could be an alternative marker for predicting CAD and severe ED could be considered an independent risk factor for CAD. Kumar et al., ${ }^{16}$ reported higher rates of multivessel CAD, diffuse CAD, and higher number of mean coronary vessels involved in CAD patients with $\mathrm{ED}$ than those without ED. ED is predictor for CAD even in the absence of cardiac symptoms. ${ }^{17}$ It is recommended that all males should be questioned for ED within the scope of assessing risk factors of CVD. ${ }^{18}$

Different prevalence rates have been reported for ED in CAD patients. Foroutan et al., ${ }^{19}$ angiographically documented that $46.4 \%$ of 401 non-diabetic male patients had ED. Sai Ravi Shanker et al., ${ }^{20}$ reported the prevalence of ED to be $47 \%$ in $180 \mathrm{CAD}$ patients, whereas Salem et al. ${ }^{15}$ determined the prevalence of ED to be $88.5 \%$ in 183 CAD patients. Kumar et al., ${ }^{16}$ reported ED in $70 \%$ of 175 patients who underwent coronary angiography. In the present study, the prevalence of ED was found to be $96.6 \%$ in the patients who underwent angiography. The reason for high prevalence of ED might be the mean age of the patients being high as $60.84 \pm 9.03$ years (range, 43-81year). In addition, in their population-based study, Akkus et al., ${ }^{21}$ reported the age-adjusted overall prevalence of ED to be $69.2 \%$ in Turkish population, of whom $33.2 \%$ had mild ED, $27.5 \%$ had moderate $\mathrm{ED}$, and $8.5 \%$ had severe ED. In multivariate analysis, they found the prevalence of moderate or severe ED to be associated with the factors such as age, residence in eastern Turkey, low educational level, unemployment, or underlying diabetes, hypertension, depression, prostate problems or lower urinary tract symptoms.

Montorsi et al., ${ }^{22}$ reported ED in $49 \%$ of 300 patients with CAD who had acute chest pain and underwent angiography. However, no significant difference was found between the patients with and without ED in terms of angiographic characteristics in that particular study. In the COBRA study ${ }^{23}$ which investigated the relationship between ED and $\mathrm{CAD}, \mathrm{ED}$ was found to be present before $\mathrm{CAD}$ in the majority by an average 2 up to 3years. Moreover, in that study, it was also reported that severity (not prevalence) of ED was associated with severity of CAD. El-sakka et al., ${ }^{24}$ conducted a study on 417 male patients, of whom $27.6 \%$ had mild ED, $30 \%$ had moderate ED, and $42.4 \%$ had severe ED. They also indicated a correlation between coronary artery risk factors and ED and reported a significant correlation between ischemic heart disease and the increased severity and progressive course of ED. Moreover, they also determined that higher degrees of ischemic heart disease were significantly associated with severe ED.

Chew et al., ${ }^{25}$ conducted a retrospective study in 1660 cases by evaluating the hospital records to identify the association between ED and consecutive cardiovascular events. They found that the incidence of cardiovascular events was significantly higher in the patients with ED. They also found a correlation between atherosclerotic cardiovascular events and ED in all patients younger than 70years old. Accordingly, they concluded that ED was a strong predictive marker for atherosclerotic CVD and that young ages in patients with ED were an even stronger predictor of cardiovascular event. Ortiz et al. ${ }^{26}$ conducted a prospective study to investigate the correlation between ED and cardiovascular events based on myocardial perfusion scintigraphy images of 287 patients. In that particular study, 137 patients $(47.8 \%)$ had ED and it was concluded that the rate of segmental myocardial perfusion and functional contraction impairment was high in the patients with ED.

In their study investigating the relationship between ED and CAD, Yaman et al., ${ }^{27}$ used coronary artery calcium (CAC) scoring, which was measured by multidetector computed tomography. In that particular study, they found a significant negative correlation between the CAC and IIEF scores. Accordingly, they concluded that CAC score increased as the severity of ED increased. On the other hand, Canat et al., ${ }^{28}$ investigated the relationship between ED and CAD in terms of number of occluded vessels and evaluated 183 patients who underwent angiography after acute myocardial infarction. They reported no difference between the patient groups with one vessel occluded, two vessels occluded, and three vessels occluded in terms of risk factors (age, waist circumference, lipid profile, diabetes, and smoking) excluding hypertension. The IIEF score was found to be significantly higher in the group with one vessel occluded as compared to the other groups. When they evaluated the effect of age, diabetes, hypertension, and smoking on the number of vessels occluded and the IIEF score, they observed that hypertension had a significant effect on ED only in patients with three-vessel occluded.

In the present study, the relationship between $\mathrm{ED}$ and $\mathrm{CAD}$ was investigated via the SS. It was found that the IIEF score significantly decreased (ED increased) as the SS, which indicates the severity of $\mathrm{CAD}$, increased. There were no significant differences between the 
SS groups in terms of the common risk factors for CAD and ED such as age, body mass index, lipid profile, presence of diabetes and hypertension, and smoking. This eliminated the confounding effect of other risk factors in the present study, in which the relationship between $\mathrm{ED}$ and $\mathrm{CAD}$ was investigated.

It is known that the prevalence of ED increases with age. ED has been reported to be a strong predictor of CVD particularly in young- and middle-aged males. ${ }^{29}$ Inman et al., ${ }^{30}$ concluded that there was a significant increase in the risk of future cardiac events in younger patients with ED, whereas ED had a little prognostic importance in older patients. In another study conducted in $\geq 65$-yearold CAD patients, Justo et al., ${ }^{31}$ concluded that lack of sexual activity and presence ED were very common in elderly men having CAD. However, the severity of ED was only related to age in sexually active males with CAD. In another study conducted to investigate the relationship between $\mathrm{ED}$ and $\mathrm{CAD}$, Riedner et al., ${ }^{32}$ concluded that males who were younger than 60years of age and had ED had a higher risk for chronic CAD and for severer diseases diagnosed by coronary angiography. In the present study, when we investigated the effect of age on the IIEF score in the SS groups, we found that the IIEF score was significantly higher in the low SS group as compared to those in the moderate and high SS groups in 43-59-year age group. Although this was similar in the other age groups, a statistically significant difference could not be demonstrated due to the limited number of patients.

The incidence of ED is high in diabetic patients. ${ }^{33}$ It has also been reported that the risk of CAD is higher in diabetic patients with $\mathrm{ED}$ than those without ED. ${ }^{29,34}$ In their study conducted on type 2 diabetic patients $(\mathrm{n}=50)$ without overt CVD, Meena et al. ${ }^{35}$ reported the prevalence of ED to be $78 \%$. Moreover, they found that the patients with ED had higher cardiovascular risk than those without ED and that 10years coronary, cardiac, vascular, and atherosclerotic risks were also higher in the patients with ED. Accordingly, they concluded that when ED severity increased, total cardiovascular risk was also significantly increased in diabetic patients. In another prospective study conducted on 6304 diabetic patients, Batty et al. ${ }^{36}$ investigated the relationship between ED and CVD related mortality and they reported that ED patients were at higher risk for CVD (hazard ratio: 1.19 and 95\% CI: 1.08-1.32) and for CAD (hazard ratio: 1.35 and 95\% CI: 1.16-1.56). It was concluded in that particular study that there was a relationship between ED and a wide range of cardiovascular diseases in males with diabetes mellitus. However, in the present study, no statistically significant difference was determined between the IIEF scores of the patients with and without diabetes among the SS groups.

Hypertension is another risk factor for ED. The prevalence of ED according to the IEEF-5 score is reported to be $67 \%$ in hypertensive patients. ${ }^{37}$ Cordeiro et al. ${ }^{38}$ conducted a study in 263 hypertensive patients and demonstrated that the presence of ED was associated with the increased risk of CAD. On the other hand, in the present study, no statistically significant difference was found between the IIEF scores of the patients with and without hypertension among the SS groups. Studies have demonstrated the relationship between smoking and ED. ${ }^{39,40}$ Both the number of cigarettes and duration of smoking have an influence on ED. In the present study, no statistically significant difference was determined between the IIEF scores of smokers and non-smokers among the SS groups.

\section{Conclusion}

In conclusion, the present study demonstrated that degree of ED increased with the increase in the SS, which indicates the severity of CAD. Early diagnosis of ED provides an opportunity to assess cardiovascular risk factors, to take necessary measures, and to prevent future cardiovascular events even a patient is asymptomatic. Detection of the SS was also found beneficial in demonstrating the severity of $\mathrm{CAD}$ and ED.

\section{Acknowledgments}

None.

\section{Conflicts of interest}

Author declares there are no conflicts of interest.

\section{Funding}

None.

\section{References}

1. Vlachopoulos C, Jackson G, Stefanadis C, et al. Erectile dysfunction in the cardiovascular patient. Eur Heart J . 2013;34(27):2034-2046.

2. Gaziano TA. Cardiovascular disease in the developing world and its cost-effective management. Circulation. 2005;112(23):3547-3553.

3. Nascimento ER, Maia AC, Pereira V, et al. Sexual dysfunction and cardiovascular diseases: a systematic review of prevalence. Clinics (Sao Paulo). 2013;68(11):1462-1468.

4. Hatzimouratidis K, Amar E, Eardley I, et al. Guidelines on Male Sexual Dysfunction: Erectile dysfunction and premature ejaculation. Eur Urol 2010;57(5):804-814.

5. Caskurlu T, Tasci AI, Resim S, et al. The etiology of erectile dysfunction and contributing factors in different age groups in Turkey. Int J Urol. 2004;11((7):525-529.

6. Ewane KA, Lin HC, Wang R. Should patients with erectile dysfunction be evaluated for cardiovascular disease? Asian J Androl. 2012;14(1):138-144.

7. Nehra A, Jackson G, Miner M, et al. The Princeton III Consensus recommendations for the management of erectile dysfunction and cardiovascular disease. Mayo Clin Proc . 2012;87(8):766-778.

8. Serruys PW, Morice MC, Kappetein AP, et al. Percutaneous coronary intervention versus coronary-artery bypass grafting for severe coronary artery disease. N Engl J Med. 2009;360:961-972.

9. ChakrabartiAK, Gibson CM. The SYNTAX score: usefulness, limitations, and future directions. J Invasive Cardiol. 2011;23(12):511-512.

10. Rosen RC, Riley A, Wagner G, et al. The international index of erectile function (IIEF): a multidimensional scale for assessment of erectile dysfunction. Urology. 1997;49(6):822-830.

11. Turunç T, Deveci S, Güvel S, et al. The assessment of Turkish validation with 5 question version of international index of erectile function (IIEF5). Turkish J Urology. 2007;33(1):45-49.

12. Shin D, Pregenzer G Jr, Gardin JM. Erectile dysfunction: a disease marker for cardiovascular disease. Cardiol Rev. 2011;19(1):5-11.

13. Meller SM, Stilp E, Walker CN, et al. The link between vasculogenic erectile dysfunction, coronary artery disease, and peripheral artery disease: role of metabolic factors and endovascular therapy. $J$ Invasive Cardiol . 2013;25(6):313-319.

14. Gandaglia G, Briganti A, Jackson G, et al. A systematic review of the association between erectile dysfunction and cardiovascular disease. Eur Urol . 2014;65(5):968-978.

15. Salem S, Abdi S, Mehrsai A, et al. Erectile dysfunction severity as a risk predictor for coronary artery disease. J Sex Med . 2009;6(12):3425-3432. 
16. Kumar J, Bhatia T, Kapoor A, et al. Erectile dysfunction precedes and is associated with severity of coronary artery disease among Asian Indians. $J$ Sex Med . 2013;10(5):1372-1379.

17. Jackson G. Erectile dysfunction and asymptomatic coronary artery disease: frequently detected by computed tomography coronary angiography but not by exercise electrocardiography. Int J Clin Pract . 2013;67(11):1159-1162.

18. Jackson G, Nehra A, Miner M, et al. The assessment of vascular risk in men with erectile dysfunction: the role of the cardiologist and general physician. Int J Clin Pract . 2013;67(11):1163-1172.

19. Foroutan SK, Rajabi M. Erectile dysfunction in men with angiographically documented coronary artery disease. Urol J. 2007;4(1):28-32.

20. Sai Ravi Shanker A, Phanikrishna B, Bhaktha Vatsala Reddy C. Association between erectile dysfunction and coronary artery disease and its severity. Indian Heart J . 2013;65(2):180-186.

21. Akkus E, Kadioglu A, Esen A, et al. Turkish Erectile Dysfunction Prevalence Study Group. Prevalence and correlates of erectile dysfunction in Turkey: a population-based study. Eur Urol. 2002;41(3):298-304.

22. Montorsi F, Briganti A, Salonia A, et al. Erectile dysfunction prevalence, time of onset and association with risk factors in 300 consecutive patients with acute chest pain and angiographically documented coronary artery disease. Eur Urol. 2003;44:360-364.

23. Montorsi P, Ravagnani PM, Galli S, et al. Association between erectile dysfunction and coronary artery disease. Role of coronary clinical presentation and extent of coronary vessels involvement: the COBRA trial. Eur Heart J. 2006;27(22):2632-2639.

24. El-Sakka AI, Morsy AM, Fagih BI, et al. Coronary artery risk factors in patients with erectile dysfunction. J Urol. 2004;172(1):251-254.

25. Chew KK, Finn J, Stuckey B, et al. Erectile dysfunction as a predictor for subsequent atherosclerotic cardiovascular events: findings from a linked-data study. J Sex Med. 2010;7(1):192-202.

26. Ortiz J, Ortiz ST, Monaco CG, et al. Erectile dysfunction: marker for myocardial perfusion impairment? Arq Bras Cardiol. 2005;85(4):241-246.

27. Yaman O, Gulpinar O, Hasan T, et al. Erectile dysfunction may predict coronary artery disease: relationship between coronary artery calcium scoring and erectile dysfunction severity. Int Urol Nephrol. 2008;40(1):117-123.

28. Canat L, Cicek G, Atis G, et al. Is there a relationship between severity of coronary artery disease and severity of erectile dysfunction? Int Braz J Urol . 2013;39(4):465-473.
29. Miner M, Seftel AD, Nehra A, et al. Prognostic utility of erectile dysfunction for cardiovascular disease in younger men and those with diabetes. Am Heart J. 2012;164(1):21-28.

30. Inman BA, Sauver JL, Jacobson DJ, et al. A population-based, longitudinal study of erectile dysfunction and future coronary artery disease. Mayo Clin Proc. 2009;84(2):108-113.

31. Justo D, Arbel Y, Mulat B, et al. Sexual activity and erectile dysfunction in elderly men with angiographically documented coronary artery disease. International Journal of Impotence Research. 2010;22(1):40-44.

32. Riedner CE, Rhoden EL, Fuchs SC, et al. Erectile dysfunction and coronary artery disease: an association of higher risk in younger men. $J$ Sex Med . 2011;8(5):1445-1453.

33. Phé V, Rouprêt M. Erectile dysfunction and diabetes: a review of the current evidence-based medicine and a synthesis of the main available therapies. Diabetes Metab. 2012;38(1):1-13.

34. Gandaglia G, Salonia A, Passoni N, et al. Erectile dysfunction as a cardiovascular risk factor in patients with diabetes. Endocrine. 2013;43(2):285-292.

35. Meena BL, Kochar DK, Agarwal TD, et al. Association between erectile dysfunction and cardiovascular risk in individuals with type-2 diabetes without overt cardiovascular disease. Int J Diabetes Dev Ctries. 2009;29(4):150-154.

36. Batty GD, Li Q, Czernichow S, et al. Erectile dysfunction and later cardiovascular disease in men with type 2 diabetes: prospective cohort study based on the ADVANCE (Action in Diabetes and Vascular Disease: Preterax and Diamicron Modified-Release Controlled Evaluation) trial. J Am Coll Cardiol . 2010;56(23):1908-1913.

37. Giuliano FA, Leriche A, Jaudinot EO, et al. Prevalence of erectile dysfunction among 7689 patients with diabetes or hypertension, or both Urology. 2004;64(6):1196-1201.

38. Cordeiro AC, Mizzaci CC, Fernandes RM, et al. Simplified International Index of Erectile Function (IIEF-5) and coronary artery disease in hypertensive patients. Arq Bras Cardiol. 2012; 99(4):924-930.

39. Gades NM, Nehra A, Jacobson DJ, et al. Association between smoking and erectile dysfunction: a population-based study. Am J Epidemiol 2005;161(4):346-351.

40. Wu C, Zhang H, Gao Y, et al. The association of smoking and erectile dysfunction: results from the Fangchenggang Area Male Health and Examination Survey (FAMHES). J Androl . 2012;33(1):59-65. 